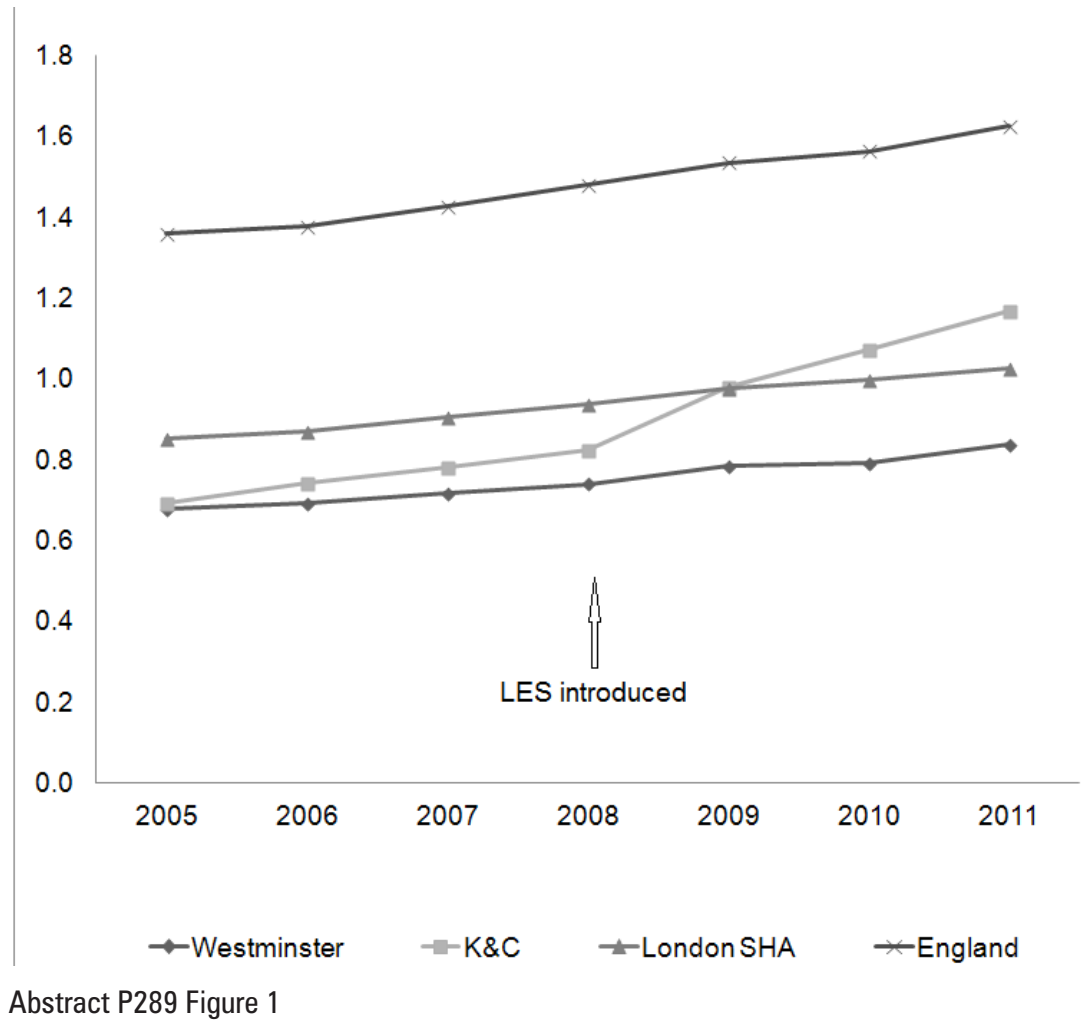

through to March 2011 obtained from the QMAS database via the NHS Information Centre. COPD prevalence data for the 4 years from the introduction of the LES (2008) were used to compare trends in COPD diagnosis between the PCT where it had been introduced, a neighbouring PCT, the London SHA and England. True COPD prevalence was estimated using data from the Health Survey for England. Diagnosed prevalence trends were extrapolated to estimate the year this level would be reached.

Results The rate of increase in COPD diagnosis with the LES remained significantly increased compared to that for London as a whole (Figure 1). Extrapolating 2008-2011 trends in prevalence, the PCT where the LES was in place would be expected to reach the modelled prevalence of COPD in 2030 whereas London as a whole would not reach the true prevalence until 2080.

Conclusion Current strategies for COPD case finding are inadequate given the scale of the problem. With appropriate incentives it is possible to achieve a sustained improvement in case-finding and such policies need to be implemented systematically.

1. Nacul L, Soljak M, Samarasundera E, Hopkinson NS, Lacerda E, Indulkar T, et al. COPD in England: a comparison of expected, model-based prevalence and observed prevalence from general practise data. J Public Health. 2011; 33(1): $108-16$.

2. Falzon C, Elkin SL, Kelly JL, Lynch F, Blake ID, Hopkinson NS. Can financial incentives for improvements in healthcare quality enhance identification of COPD in primary care? Thorax. 2011; 66(7):630.

\title{
P290 IMPACT OF A COMBINED ADMISSION AND DISCHARGE COPD CARE BUNDLE ON HOSPITAL MORTALITY AND READMISSION RATES
}

doi:10.1136/thoraxjnl-2012-202678.382

A Graham. Huddersfield Royal Infirmary, Huddersfield, UK

Background A care bundle is a group of evidence based interventions which if consistently delivered should improve outcomes.

Published work (Thorax 2012) has demonstrated the impact of a COPD discharge bundle on hospital readmission rates. There is no published information on the use of bundles to address in-hospital mortality for COPD.

Methods In April 2011 a care bundle for acute exacerbation of COPD (AECOPD) was introduced at both acute hospital sites in our Trust. The bundle content was printed on an adhesive sticker which was placed in the case notes. It consisted of a 7 point acute care section, completed on admission, and a 10 point discharge section completed prior to discharge. Multidisciplinary training and intranet guidance was provided to staff. Bundle use and compliance with each element was assessed quarterly through audit of 50 consecutive admissions. Hospital readmission rates and mortality were also reviewed.

Results Audit was undertaken in October 2011, January 2012 and April 2012. Bundle stickers were reliably placed in the medical notes (>80\%). Delivery of all 7 admission bundle elements was high in each audit $(>75 \%)$, but compliance with the timings required fell during the ongoing audit $(56 \% ; 24 \% ; 26 \%)$. Compliance was higher in patients admitted through A\&E compared to those admitted directly to MAU Compliance with all 10 discharge bundle elements initially improved but was not sustained $(26 \%$; $74 \%$; $35 \%$ ). Site specific comparison indicated that discharge bundle delivery was better with a hospital based respiratory specialist nursing "outreach" service, than with a community based "inreach" service. Overall compliance with all 17 bundle elements fell during the year (24\%; $26 \%$; $5 \%$ ). Readmission rates compared to the previous financial year fell from $14.9 \%$ to $12.8 \%$. Mortality rates also fell (figure).

Conclusion Despite the lack of full compliance with the bundle, it was well used and improved outcomes were seen in terms of mortality and readmissions. It was felt that the bundle acted as a focus for improving knowledge and delivery of COPD care. Regular training and encouragement of staff will be required to sustain compliance with use of the bundle. 


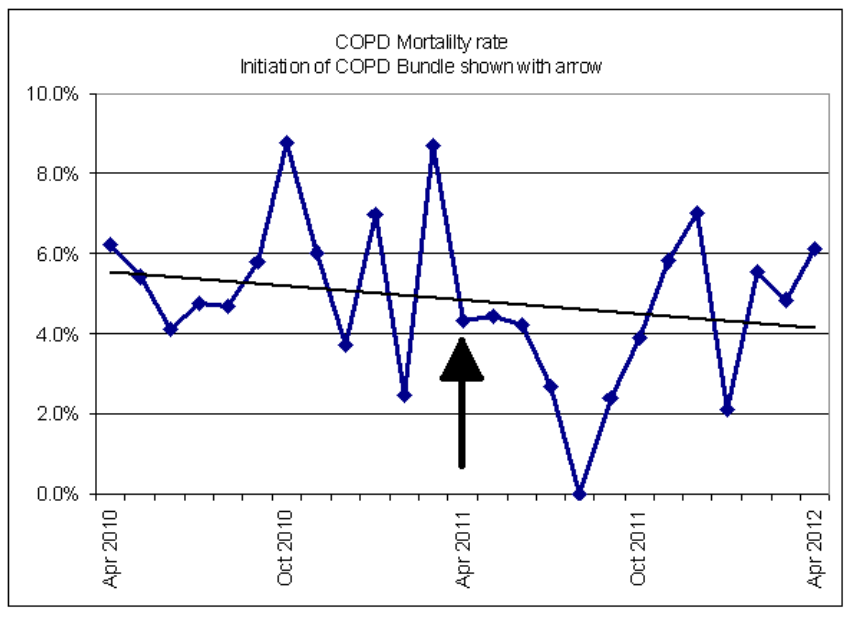

Abstract P290 Figure 1

\section{P291 COPD DISCHARGE BUNDLES: THE EXETER EXPERIENCE 2011-12}

doi:10.1136/thoraxjnl-2012-202678.383

DMG Halpin, P Batten, C Chamberlain. Royal Devon \& Exeter Hospital, Exeter, UK

Bundles which facilitate standardised quality care have been used for over 10 years (1). Recently, discharge bundles for COPD have been proposed as a way of improving outcomes, including reducing readmission rates, for patients discharged from hospital. We introduced one based on a version developed in London (2) in 2011. It comprised 5 items addressing self management, inhaler technique
(IT), smoking cessation advice (SA), pulmonary rehabilitation (PR), and follow up (FU). The bundle was completed by a respiratory nurse specialist (RNS) prior to discharge, they also planned to phone patients $24-72 \mathrm{hrs}$ post discharge.

The bundle was used in 67 patients discharged Oct 2011-Mar 2012. In total 324 patients with COPD were discharged during this time. Five patients were discharged before the bundle had been completed. It was not used if patients were confused, lived out of area, or went to intermediate or palliative care. The mean age was 72 (range 49-91), CAT score 22.5, MRC 3.66, FEV1 33\%, DOSE index 5.34 .

$58(87 \%)$ received written advice, $17(25 \%)$ were referred for or given SA, 65 (97\%) had IT checked, 21 (31\%) were referred for \& 19 (28\% declined) PR, 58(89\%) had FU requested. 39 (58\%) were phoned within 72 hrs and 60 (92\%) eventually. $77 \%$ of calls lasted 5 minutes or less. When contacted $37(57 \%)$ of patients said they were better and 52 (80\%) had no concerns. One patient was advised to restart steroids, 1 had a community matron visit arranged, 1 was readmitted \& 2 were given phone advice about coughs. One was constipated. Six of the 16 given SA were not smoking when phoned.

$12(18 \%)$ were readmitted within 30 days. This compares with a readmission rate of $27 \%$ in patients discharged during the same period who did not have the bundle completed.

Discharge bundles and follow up calls ensured this group of elderly patients with severe or very severe COPD had important aspects of their management assessed prior to discharge and this appeared to reduce readmission rates.

\section{References}

1. Burger CD, Resar RK Mayo Clinic Proceedings. 2006; 81(6):849-850

2. Hopkinson NS, et al. Thorax 2012; 67:90-2. 